\title{
3D MULTI PERSON TRACKING WITH DUAL 360 CAMERAS
}

\author{
Matthew Shere ${ }^{1}$, Hansung Kim ${ }^{1,2}$, Adrian Hilton ${ }^{1}$ \\ 1. Centre for Vision, Speech and Signal Processing \\ University of Surrey, UK \\ $\{$ m.shere, a.hilton\}@surrey.ac.uk
2. Electronics and Computer Science University of Southampton, UK h.kim@soton.ac.uk

\begin{abstract}
Person tracking is an often studied facet of computer vision, with applications in security, automated driving and entertainment. However, despite the advantages they offer, few current solutions work for $360^{\circ}$ cameras, due to projection distortion. This paper presents a simple yet robust method for 3D tracking of multiple people in a scene from a pair of $360^{\circ}$ cameras. By using 2D pose information, rather than potentially unreliable $3 \mathrm{D}$ position or repeated colour information, we create a tracker that is both appearance independent as well as capable of operating at narrow baseline. Our results demonstrate state of the art performance on $360^{\circ}$ scenes, as well as the capability to handle vertical axis rotation.
\end{abstract}

Index Terms - 360 Imaging, 360 Tracking, Panoramic Imaging, Person Tracking, Multi Person Tracking

\section{INTRODUCTION}

Beyond it's obvious security applications, human tracking in crowded scenes has a multitude of uses in the real world. Automated vehicles, safety systems and even the entertainment sector can benefit from human tracking systems. These sectors give rise to the use of wide angle and $360^{\circ}$ cameras, due to their increased visual coverage, but also introduce problems, predominantly related to non-linear image distortion. Many tracking systems are designed using perspective cameras, and often struggle with $360^{\circ}$ video due to distortions.

We present a 3D multi person tracker, capable of operating from a pair of $360^{\circ}$ cameras. The tracker is capable of operating in both indoor and outdoor environments with multiple people. Tracking is performed from 2D human pose information and a triangulated depth estimate, removing restrictions on person appearance and on any 3D pose estimates, which can be unreliable due to low $(<50 \mathrm{~cm})$ camera baselines or 2D joint mis-detections.

Our main contribution from this work is an appearanceindependent multi person tracker operating from two narrow baseline $360^{\circ}$ video sequences. This tracker estimates $3 \mathrm{D}$ po-

This work is supported by both the EPSRC (grant number EP/N 509383/1) and BBC Research and Development.
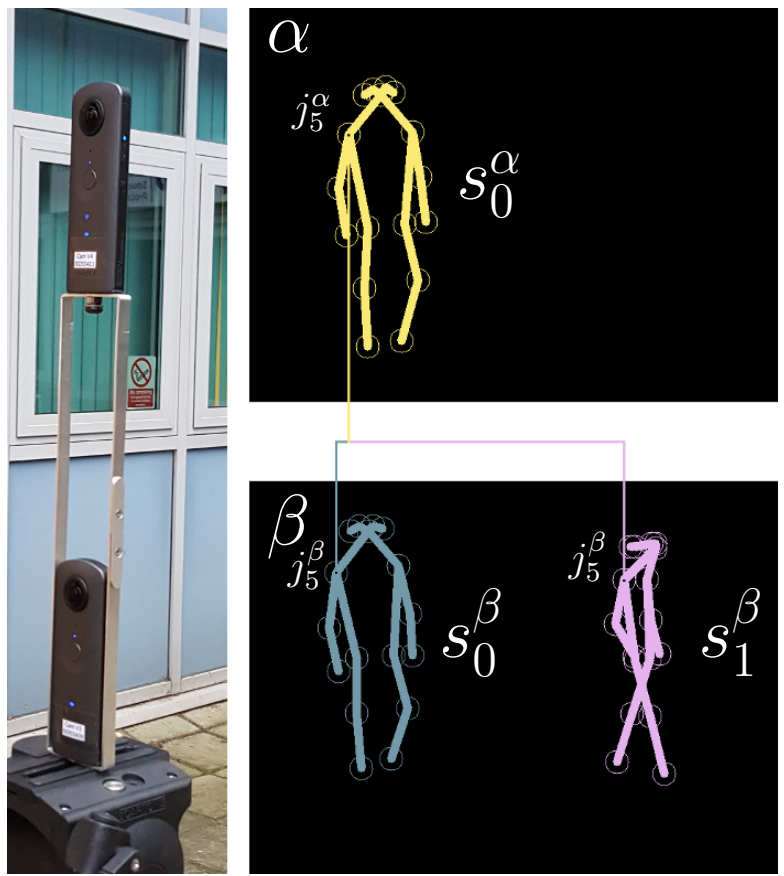

Fig. 1. Left: Our experimental setup. Right: example of our matching on a single joint. The distance between $s_{0}^{\alpha}$ and $s_{0}^{\beta}$ is significantly less than $s_{0}^{\alpha}$ and $s_{1}^{\beta}$, and is more likely to be the correct match (example using one joint only)

sition and joint locations for multiple people in indoor or outdoor scenes.

\section{RELATED WORKS}

Tracking systems associate objects identified in an initial frame over time to maintain identity throughout a video sequence. These systems need to perform re-identification[1], where tracking of the object should continue even when that object cannot be seen, reappears after occlusion, or moves into a different camera view.

Person tracking systems using handcrafted features[2, 3, $4,5]$ are pre-defined to track specific objects (such as humans in the above papers). Kahn et al.[2] exploit the relationship between cameras for tracking, while Zhou and Hoang[3] use 


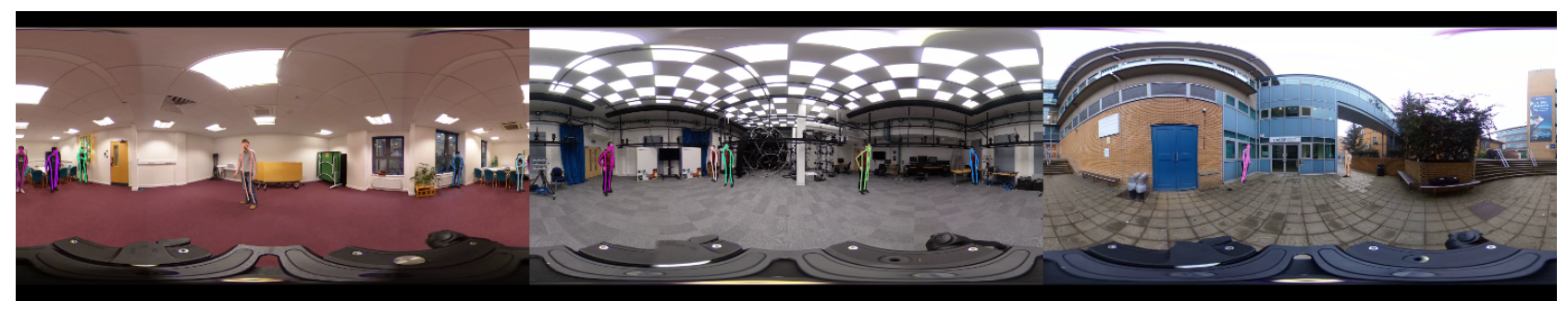

Fig. 2. Example dataset frames. Left: Random Walk (6 people). Middle: 5 Person ( 5 people). Right: Outdoor $0^{\circ}$ ( 2 people)

attributes about the person(s) being tracked, such as colour, velocity and scale. Danelljan et al. [4] jointly calculate translation and scale from a previous frame to the current one, before predicting the next frame. Finally, Zhang et al.[5] use an online Support Vector Machine and an entropy minisation function for tracking.

Deep learning has had a profound impact on tracking, as it has with other areas of Computer Vision. Nam and Han[6] created a relatively small network for object tracking, the Multi-Domain Network (MDNet). Insafutdinov et al. [7] combine deep learning approaches with more traditional methods, tracking in both a top-down and bottom-up approach. Fernando et al. [8] used lightweight Generative Adversarial Networks and an object pool to create both a short and long term memory which can then be utilised for trajectory tracking and prediction. The most recent trend of Siamese tracking networks $[9,10,11,12]$ do not track frame to frame, rather they take an exemplar image and attempt to find it within the current frame. This removes the need for special behaviours during occlusions.

While all of these tracking algorithms are effective, they all suffer from the problem that they are designed to work on perspective images, and as such were not built to deal with $360^{\circ}$ images that introduce both distortion and image wrapping[13]. $360^{\circ}$ tracking systems can overcome this, but come with their own problems. Delforouzil et al.[14] produced a generic object tracker that finds objects similar in shape and appearance to the target, then combines optical flow and trained detectors to identify the target object. Shere et al. [15] track a single individual in a multi-person scene using colour models and estimated 3D pose, with information shared between two $360^{\circ}$ cameras. Both of these methods are limited to a single person and cannot be used when appearance is similar (such as in team sports).

\section{METHOD}

Our tracking method is split into two major components, spatial correspondence, where we attempt to match people between two frames at the same time from two separate views, and temporal matching, where we match our paired people to the collection of existing pairs we have from the previous frame. In both cases, we use a pair of cameras in a verti- cal stack (see Figure 1, left) and with inter-camera rotations minimised. As such, any given detail in one image should be directly above/below the corresponding detail in the other image. We exploit this relationship to track each person.

\subsection{Spatial Correspondence}

We perform our human tracking using a pair of $360^{\circ}$ cameras, $\alpha, \beta$, that are vertically aligned and whose extrinsics are known. From these, we obtain a series of video frames $\left\{f_{n}^{\alpha}\right.$, $\left.f_{n}^{\beta}\right\}$, where $n=\{1,2 \ldots N\}$ is the frame number and $N$ is the total length of the video. Each frame is of size $h, w$, representing image height and width respectively.

For each frame $f_{n}^{c}$, we obtain a set of 2D pose estimates[16] $S_{n}^{c}$, where $c=\{\alpha, \beta\}$ is the camera. Note we only obtain pose estimates central half of the image, $\frac{h}{4}$ to $\frac{3 h}{4}$, to minimise distortion problems. This set contains 0 or more pose estimates $s_{i} \in S_{n}^{c}$, where $s$ is the skeletal pose estimate and $i$ is the index of the skeleton in $S_{n}^{c}$. These pose estimates each contain a joints $j_{o}^{c} \in s_{i}^{c}$, where $o$ is the joint number. Each joint $j$ consists of $(x, y)$ coordinate data and a confidence value $\kappa$, such that $j_{o}^{c}=\left(x_{o}^{c}, y_{o}^{c}, \kappa_{o}^{c}\right)$

Initially, we wish to solve a matching problem of $S_{0}^{\alpha}, S_{0}^{\beta}$. To achieve this, we measure the horizontal distance between each $s_{i}^{\alpha} \in S_{0}^{\alpha}, s_{k}^{\beta} \in S_{0}^{\beta}$ (Figure 1, right). This distance is defined in eq. 1

$$
\operatorname{dist}\left(s_{i}^{\alpha}, s_{k}^{\beta}\right)=\sum_{o=0}^{O} \mu \sigma\left(j_{o}^{\alpha}, j_{o}^{\beta}\right)
$$

where $O$ is the total number of joints, $\mu$ is a weight and

$$
\begin{aligned}
\sigma_{1} & =\left|x_{o}^{\alpha}-x_{o}^{\beta}\right| \\
\sigma_{2} & =\sigma_{1}-w \\
\sigma\left(j_{o}^{\alpha}, j_{o}^{\beta}\right) & =\min \left(\sigma_{1}, \sigma_{2}\right)
\end{aligned}
$$

is the shortest circular distance between a pair of $x$ coordinates.

From this, we generate a cost matrix $C$, containing the $\operatorname{cost} \operatorname{dist}\left(s_{i}^{\alpha}, s_{k}^{\beta}\right)$ for each pair of skeletons in $s_{i}^{\alpha} \in S_{n}^{\alpha}, s_{k}^{\beta} \in$ $S_{n}^{\beta}$. We use the Hungarian algorithm[17] to obtain the lowest cost pairings. Since our cameras are vertically aligned, and therefore skeletons should also be vertically aligned, we remove any pairings where the confidence weighted centroids 

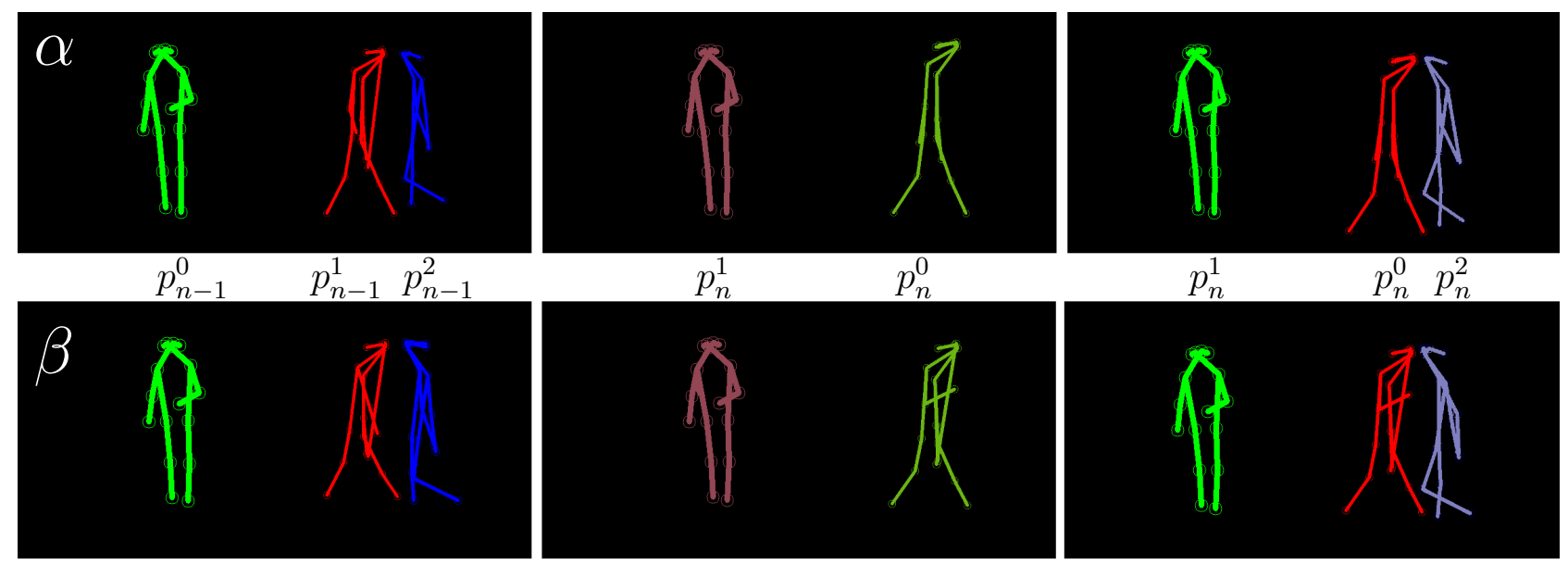

Fig. 3. Example of our matching across two frames. $p_{n-1}^{0}$ is matched with $p_{n}^{1}$ using the Hungarian method, but the cost of matching either $p_{n-1}^{1}$ or $p_{n-1}^{2}$ to $p_{n}^{0}$ are similar, so $p_{n-1}^{1}$ is matched to $p_{n}^{0}$ using depth, and $p_{n-1}^{2}$ is moved by it's velocity

are further apart than $\Delta_{p}$ in the horizontal plane, where $\Delta_{p}=$ $20^{\circ}$ was empirically found to work well. This confidence weighted centroid is calculated as

$$
c w c\left(s_{i}^{c}, \Theta\right)=\frac{\sum_{o \in \Theta}\left[x_{o}^{c}, y_{o}^{c}\right]^{T} * \kappa_{o}^{c}}{\sum_{o \in \Theta} \kappa_{o}^{c}} \quad \text { for } j_{o}^{c} \in s_{i}^{c}
$$

where $\Theta$ is the set of joint indices used for calculation. In our case, we use the left and right shoulder joints.

For each $s_{i}^{\alpha} \in S_{n}^{\alpha}, s_{k}^{\beta} \in S_{n}^{\beta}$ paired in this way, we generate a skeleton pair $p_{n}^{i}=\left\{s_{i}^{\alpha}, s_{k}^{\beta}\right\}$. These skeleton pairs then form a set $p_{n}^{i} \in P_{n}$, which are then used for temporal matching.

\subsection{Temporal Matching}

Our next step for frame $n>0$ is to match our set of skeleton pairs $P_{n}$ to those of the previous frame $P_{n-1}$. For each $p_{i} \in$ $P_{N}, p_{k} P_{n-1}$, we construct a cost matrix $M$ by taking the difference of the pairs of skeletons, such that

$$
\begin{gathered}
\operatorname{mdist}\left(p_{i}, p_{k}\right)=\operatorname{dist}\left(s_{i}^{\alpha}, s_{k}^{\alpha}\right)+\operatorname{dist}\left(s_{i}^{\beta}, s_{k}^{\beta}\right) \\
\operatorname{for}\left\{s_{i}^{\alpha}, s_{i}^{\beta}\right\} \in p_{n}^{i},\left\{s_{k}^{\alpha}, s_{k}^{\beta}\right\} \in p_{n}^{k}
\end{gathered}
$$

where $\mu=\max \left(\kappa_{o}^{\alpha}, \kappa_{o}^{\beta}\right)$. This is done both to exclude low confidence joints from overly influencing the cost, and also to increase the chance of matching with people aligned in the same direction.

We again use the Hungarian algorithm on the cost matrix $M$ to obtain a set of minimum cost matches. This is supplemented with depth matching where the cost of non selected matches are within $\lambda$ of the selected match, in order to better tackle occlusions. Finally, we remove any matches where the confidence weighted centroids are further apart than $\Delta_{m}$. We empirically found that $\lambda=20 \%, \Delta_{m}=40^{\circ}$ provided good results across our datasets.

\begin{tabular}{|l|c|c|c|}
\hline Dataset & SiamRPN++ & Shere et al. & Proposed \\
\hline \hline Cross Walk & $90.13 \%$ & $78.84 \%$ & $\mathbf{1 0 0 . 0 0 \%}$ \\
Go Around & $97.21 \%$ & $82.58 \%$ & $\mathbf{1 0 0 . 0 0 \%}$ \\
Pair Loop & $59.86 \%$ & $49.75 \%$ & $\mathbf{9 9 . 9 6 \%}$ \\
Random Walk & $59.84 \%$ & $66.91 \%$ & $\mathbf{9 4 . 2 8 \%}$ \\
\hline Outdoor 0 & $99.71 \%$ & $64.81 \%$ & $\mathbf{1 0 0 . 0 0 \%}$ \\
${\text { Outdoor } 40^{\circ}}^{\circ}$ & $99.23 \%$ & $64.81 \%$ & $\mathbf{1 0 0 . 0 0 \%}$ \\
Outdoor $80^{\circ}$ & $57.40 \%$ & $92.79 \%$ & $\mathbf{1 0 0 . 0 0 \%}$ \\
\hline 5 Person & $70.39 \%$ & $62.86 \%$ & $\mathbf{9 9 . 2 9 \%}$ \\
\hline
\end{tabular}

Table 1. Performance of the proposed algorithm

Each matched member of $P_{n-1}$ is assigned a velocity calculated as the average weighted centroid movement of the member skeletons between $P_{n-1}$ and $P_{n}$, while joints of skeletons belonging to non-matched members of $P_{n-1}$ are moved by their velocity, then added into $P_{n}$. This provides a simple but effective way of tracking individuals through occlusions (Figure 3).

\section{EXPERIMENTS}

We evaluate our system on several datasets, comprising of multi person scenes in both indoor and outdoor environments. Each scene is filmed using a pair of Ricoh Theta $\mathrm{V}$ cameras[18] with a baseline of $30 \mathrm{~cm}$ between cameras. We evaluate our algorithm against both SiamRPN++[12], a state of the art siamese tracker designed for a monocular perspective video sequence, and Shere et al. [15], a handcrafted appearance based tracker using stereo $360^{\circ}$ video sequences.

\subsection{Tracking Experiment}

The first experiment was performed on a series of indoor datasets, covering both simple and difficult scenes. Cross Walk is a 4 person scene with 2 people crossing each other 


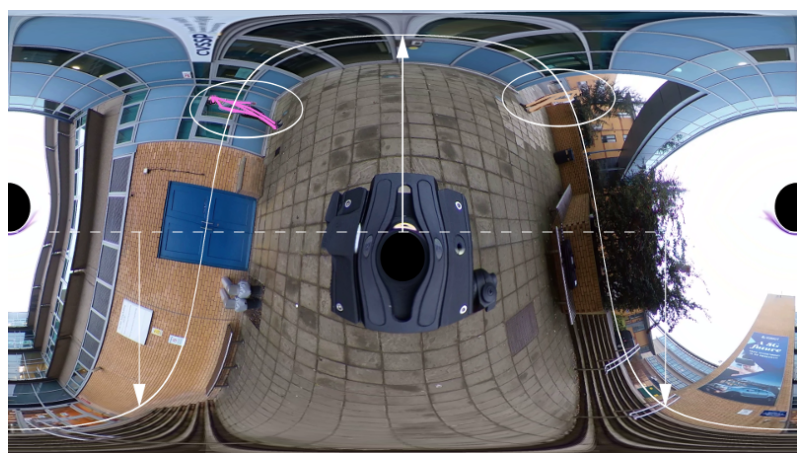

Fig. 4. An example frame from Outdoor $80^{\circ}$. Skeletons are overlaid and circled. The solid white line is the pre-rotation horizon, while the arrows show the movement of the horizon.

in front of the camera. Go Around is a 5 person scene, with 1 person circling around a second person. Double Loop is a 3 person scene, with 2 people looping around the camera in opposite directions. Random Walk is a 6 person scene with all people moving randomly in the scene. All of these scenes were captured in an indoor non-studio environment. Any individuals outside of the capture area are expressly not tracked, even if they are visible in the scene.

The upper section of Table 1 details our results. Using only $2 \mathrm{D}$ pose information and estimated depth, we outperform SiamRPN++, even on Cross Walk and Go Around, neither of which have people moving "behind" the camera (i.e. moving off one side of the frame and moving onto the other). Shere et al.meanwhile struggles with the vertical (rather than horizontal) configuration of the cameras, causing problems with the appearance matching.

\subsection{Outdoor Experiment}

The second experiment was performed on an series of outdoor datasets, covering a simple scene designed to test the tracker on both outdoor lighting conditions and $y$ axis rotation. The scene is a 2 person scene where the people cross each other in front of the camera. This dataset is then rotated on the $y$ axis by $-40^{\circ}$ and $-80^{\circ}$ (see Figure 4 ), along with any segmentation or keypoint information.

The second section of Table 1 details our results. As we can see, our method is able to handle the rotation of the image, as long as keypoints are accurate. SiamRPN++ performs well at lower rotation, but image distortion quickly makes tracking unstable at higher rotation. Shere et al.increase performance at high rotation, although the tracking produced is unstable, particularly around the occlusion points.

\subsection{Appearance Experiment}

The third experiment was performed on indoor synthetic dataset designed to demonstrate the trackers performance in similar appearance situations. The same individual was

\begin{tabular}{|l|c|c|c|}
\hline Person & SiamRPN++ & Shere et al. & Proposed \\
\hline \hline Person 1 & $83.50 \%$ & $70.20 \%$ & $\mathbf{9 9 . 1 4 \%}$ \\
Person 2 & $56.65 \%$ & $23.89 \%$ & $\mathbf{9 8 . 4 0 \%}$ \\
Person 3 & $99.88 \%$ & $\mathbf{1 0 0 . 0 0 \%}$ & $99.01 \%$ \\
Person 4 & $29.19 \%$ & $41.13 \%$ & $\mathbf{1 0 0 . 0 0 \%}$ \\
Person 5 & $82.76 \%$ & $49.26 \%$ & $\mathbf{9 9 . 8 8 \%}$ \\
\hline
\end{tabular}

Table 2. Breakdown of tracking results on 5 Person, the numbers relate to the accuracies on each individual person

\begin{tabular}{|l|c|c|c|}
\hline Technique & Runs & Secs/frame & Total secs/frame \\
\hline \hline SiamRPN++ & 10 & $\mathbf{0 . 8 9}$ & 8.9 \\
Shere et al. & 5 & 92.43 & 462.15 \\
Proposed & $\mathbf{1}$ & 1.19 & $\mathbf{1 . 1 9}$ \\
\hline
\end{tabular}

Table 3. Runs required and average execution time per frame for a single run on 5 Person. SiamRPN++ is fastest per run, but requires multiple runs on each dataset.

recorded 5 times performing different movements to produce a composite scene containing occlusions caused by an individual of highly similar appearance. The final section of Table 1 details our results, which show that by not using any appearance information, we become highly robust to scenes containing similar individuals. Table 2 goes into more detail of the relative performance on the 5 people. Note that only persons 1 and 2 go "behind" the camera, the persons 3, 4 and 5 all stay in frame as would be expected on a perspective camera, experiencing only occlusions.

\subsection{Performance Experiment}

Finally, we measured the execution time of each system using an Intel Core i7-8700 CPU, 64GB of RAM and a GeForce GTX 1080 Ti GPU. We evaluated on the 5 Person dataset, with the results in Table 3. Note that SiamRPN++ needed 10 runs total (5 people across 2 cameras), while Shere et al. needed 5 runs (5 people). In contrast, the proposed system only required one run.

\section{CONCLUSIONS}

We have presented a novel two camera, multi person tracking algorithm, capable of both handling the representation challenges of $360^{\circ}$ video, as well as being able to track multiple people simultaneously. We produce state of the art results in tasks including similar appearance, and those including large pitch changes. We achieve this without sacrificing either execution time or accuracy from appearance based methods, although our low baseline shows limitations during multiple occlusions, due to calculated depth inaccuracy and drift. As such, a future work direction would be to include appearance information to supplement depth information on close proximity cases, or to expand the camera baseline to produce more accurate depth estimates. 


\section{REFERENCES}

[1] M. Farenzena, L. Bazzani, A. Perina, V. Murino, and M. Cristani, "Person re-identification by symmetrydriven accumulation of local features," in 2010 IEEE Computer Society Conference on Computer Vision and Pattern Recognition, June 2010, pp. 2360-2367.

[2] S. Khan, O. Javed, Z. Rasheed, and M. Shah, "Human tracking in multiple cameras," in Proceedings Eighth IEEE International Conference on Computer Vision. ICCV 2001, July 2001, vol. 1, pp. 331-336 vol.1.

[3] Jianpeng Zhou and Jack Hoang, "Real time robust human detection and tracking system," in 2005 IEEE Computer Society Conference on Computer Vision and Pattern Recognition (CVPR'05) - Workshops, Sep. 2005, pp. 149-149.

[4] Martin Danelljan, Gustav Häger, Fahad Shahbaz Khan, and Michael Felsberg, "Accurate scale estimation for robust visual tracking," in Proceedings of the British Machine Vision Conference. 2014, BMVA Press.

[5] Jianming Zhang, Shugao Ma, and Stan Sclaroff, "Meem: Robust tracking via multiple experts using entropy minimization," in Computer Vision - ECCV 2014, David Fleet, Tomas Pajdla, Bernt Schiele, and Tinne Tuytelaars, Eds., Cham, 2014, pp. 188-203, Springer International Publishing.

[6] Hyeonseob Nam and Bohyung Han, "Learning multidomain convolutional neural networks for visual tracking," in The IEEE Conference on Computer Vision and Pattern Recognition (CVPR), June 2016.

[7] Eldar Insafutdinov, Mykhaylo Andriluka, Leonid Pishchulin, Siyu Tang, Evgeny Levinkov, Bjoern Andres, and Bernt Schiele, "Arttrack: Articulated multiperson tracking in the wild," in IEEE Conference on Computer Vision and Pattern Recognition (CVPR). IEEE, 2017, vol. 4327.

[8] T. Fernando, S. Denman, S. Sridharan, and C. Fookes, "Tracking by prediction: A deep generative model for mutli-person localisation and tracking," in Proceedings - 2018 IEEE Winter Conference on Applications of Computer Vision, WACV 2018, 2018, vol. 2018-January, pp. 1122-1132.

[9] Luca Bertinetto, Jack Valmadre, João F. Henriques, Andrea Vedaldi, and Philip H. S. Torr, "Fully-convolutional siamese networks for object tracking," in Computer Vision - ECCV 2016 Workshops, Gang Hua and Hervé Jégou, Eds., Cham, 2016, pp. 850-865, Springer International Publishing.
[10] B. Li, J. Yan, W. Wu, Z. Zhu, and X. Hu, "High performance visual tracking with siamese region proposal network," in Proceedings of the IEEE Computer Society Conference on Computer Vision and Pattern Recognition, 2018, pp. 8971-8980.

[11] Qiang Wang, Li Zhang, Luca Bertinetto, Weiming $\mathrm{Hu}$, and Philip HS Torr, "Fast online object tracking and segmentation: A unifying approach," in Proceedings of the IEEE Conference on Computer Vision and Pattern Recognition, 2019.

[12] Bo Li, Wei Wu, Qiang Wang, Fangyi Zhang, Junliang Xing, and Junjie Yan, "Siamrpn++: Evolution of siamese visual tracking with very deep networks," in The IEEE Conference on Computer Vision and Pattern Recognition (CVPR), June 2019.

[13] T.-W. Mi and M.-T. Yang, “Comparison of tracking techniques on 360-degree videos," Applied Sciences (Switzerland), vol. 9, no. 16, 2019.

[14] A. Delforouzi, S.A.H. Tabatabaei, K. Shirahama, and M. Grzegorzek, "Unknown object tracking in 360degree camera images," in Proceedings - International Conference on Pattern Recognition, 2016, vol. 0, pp. 1798-1803.

[15] Matthew Shere, Hansung Kim, and Adrian Hilton, "3d human pose estimation from multi person stereo 360 scenes," in The IEEE Conference on Computer Vision and Pattern Recognition (CVPR) Workshops, June 2019.

[16] Jiefeng Li, Can Wang, Hao Zhu, Yihuan Mao, Hao-Shu Fang, and Cewu Lu, "Crowdpose: Efficient crowded scenes pose estimation and a new benchmark," in The IEEE Conference on Computer Vision and Pattern Recognition (CVPR), June 2019.

[17] Harold W Kuhn, "The hungarian method for the assignment problem," Naval research logistics quarterly, vol. 2, no. 1-2, pp. 83-97, 1955.

[18] "Ricoh Theta V," https://theta360.com/uk/about/theta/v.html, 2019, Accessed: 2019-02-22. 